\title{
Differentially expressed genes in longitudinal axis of the fox's hippocampus
}

\author{
Yury Alexandrovich \\ ICG SB RAS, Novosibirsk, Russia \\ alexandrovich@bionet.nsc.ru
}

\author{
Larisa Meister \\ ICG SB RAS, Novosibirsk, Russia \\ meister@bionet.nsc.ru
}

Yury Herbeck

ICG SB RAS, Novosibirsk, Russia

herbek@bionet.nsc.ru

\begin{abstract}
The role of hippocampus in varied neural processes is well known; however, hippocampus is not a uniform structure. Our results demonstrated differences in gene expression between dorsal and ventral hippocampus in foxes. The expression of $C Y P 26 B 1$ in dorsal hippocampus is increased in tame foxes relatively to aggressive ones.
\end{abstract}

Keywords - dorsal hippocampus, ventral hippocampus, adult neurogenesis

\section{Motivation and aim}

\section{Motivation}

Hippocampus is one of the brain structures that regulate stress, emotions, memory and cognitive functions. Moreover, hippocampus is also responsible for adult neurogenesis. It has been shown previously that tame foxes have decreased stress-response and increased adult neurogenesis in hippocampus [1,2]. Stress-response and neurogenesis may be interrelated $[3,4]$. However, when searching for the molecular mechanisms of these processes and their interrelation in hippocampus, it is important to consider that dorsal and ventral hippocampus are functionally distinct structures with its longitudinal axis being organized along a gradient $[5,6]$. Particularly, it is often supposed that ventral hippocampus is responsible for stress and emotions, while dorsal hippocampus plays its role in cognitive functions and memory.

Aim

The aim of this study was to test whether hippocampal longitudinal differences by gene expression were present in red foxes (Vulpes vulpes) and to compare the expression of genes between tame and aggressive foxes.

\section{Methods}

For the analysis dorsal and ventral hippocampus tissues of male foxes (7-8 months old) were used. Gene expression was evaluated using RT-qPCR analysis. CANX gene was used as a reference gene. Mann-Whitney non-parametric test was used for pair-wise comparison.

\section{Results}

Of the genes studied, the gene expression in dorsal hippocampus is increased relatively to ventral hippocampus for $C Y P 26 B 1, C A D M 2$ and $K C N D 2$ genes. Conversely, in ventral hippocampus the gene expression in increased relatively to dorsal hippocampus for NTS, NR2F2, ADRA1A, $T R H R$, and CPNE2 genes (all cases $\mathrm{p}<0.001$ ). No significant difference was found for $K C N D 3$ gene.

In addition, the expression of CYP26B1 gene was demonstrated to be increased significantly in the dorsal hippocampus in tame foxes relatively to aggressive ones $(\mathrm{p}<0.01)$.

The data obtained conform to the results shown for rodents except for the two genes: NTS gene expression is increased in ventral hippocampus in foxes but in dorsal hippocampus in rats [7] and KCND3 gene expression difference is demonstrated in mice but not in foxes [8].

CYP26B1 is an enzyme responsible for degradation of retinoic acid, which is one of the important participants in the neurogenesis process. The found difference in CYP26B1 gene expression in dorsal hippocampus indicates the role of retinoic acid pathway in the mechanism of elevation of adult neurogenesis in tame foxes. However, the absence of this difference in the ventral hippocampus indicates, perhaps, more complex causes of changes in neurogenesis.

\section{ACKNOWLEDGMENT}

Supported by the RSF (19-74-10041).

\section{References}

[1] Trut L. et al. (2009) Animal evolution during domestication: the domesticated fox as a model. Bioessays. 31(3):349-360.

[2] Huang S. et al. (2015) Selection for tameness, a key behavioral trait of domestication, increases adult hippocampal neurogenesis in foxes. Hippocampus. 25(8):963-975.

[3] Sánchez-Vidaña D. I. et al. (2016) Repeated treatment with oxytocin promotes hippocampal cell proliferation, dendritic maturation and affects socio-emotional behavior. Neuroscience. 333:65-77.

[4] Song J. et al. (2012) Modification of hippocampal circuitry by adult neurogenesis. Developmental Neurobiology. 72:1032-1043.

[5] Fanselow M. S., Dong H. W. (2010). Are the dorsal and ventral hippocampus functionally distinct structures? Neuron. 65(1):7-19.

[6] Strange B. A. et al. (2014). Functional organization of the hippocampal longitudinal axis. Nature Reviews Neuroscience. 15(10):655-669.

[7] Lee A. R. et al. (2017). Dorsal and ventral hippocampus differentiate in functional pathways and differentially associate with neurological disease-related genes during postnatal development. Frontiers in molecular neuroscience. 10:331.

[8] Cembrowski M. S. et al. (2016). Hipposeq: a comprehensive RNA-seq database of gene expression in hippocampal principal neurons. Elife. 5:e14997. 